\title{
浅谈上海浦江线 RT21 综合监控系统
}

\section{Talking about the RT21 Integrated Monitoring System of Shanghai Pujiang Line}

\author{
华佳鸣 \\ Jiaming Hua \\ 上海申凯公共交通运营管理有限公司 中国・上海 200070 \\ Shanghai Keolis Public Transport Operation Management Co.,Ltd., Shanghai, 200070, China
}

\begin{abstract}
摘 要: 上海浦江线 RT21 综合监控系统平台在浦江线运营中起到了相当大的辅助作用,其把各自无直接关联且相对独立开 来的许多专业与下属车站和控制中心凝结为一个运行整体, 达成浦江线各系统与专业间的资源整合与信息联动, 从而增强了 浦江线主体应对各类突发事件的应变能力与反应速度, 大幅度提高了浦江线整体运营管理水平。由于综合监控系统在上海轨 道交通入网线路中使用度相对并不高,在浦江线之前也仅 10 号线投用,特此开展浦江线 RT2 1 综合监控系统研究,一方面深 入探讨浦江线 RT2 1 综合监控系统的应用架构与特点,另一方面为以后上海轨道交通入网线路投用此类综合监控系统奠定良 好的技术基础。
\end{abstract}

\begin{abstract}
The RT21 integrated monitoring system platform of Shanghai Pujiang line has played a considerable auxiliary role in the operation of Pujiang line. It condenses many disciplines that are not directly related and relatively independent with the subordinate stations and control centers into a whole operation. The integration of resources and information linkages between various systems and disciplines has enhanced, the Pujiang line's main body's ability to respond to various emergencies and response speed, and greatly improved the overall operation and management level of the Pujiang line. Since the integrated monitoring system is relatively not used in Shanghai rail transit network lines, and only line 10 was put into use before the Pujiang line, the research on the Pujiang line RT21 integrated monitoring system is hereby carried out, and on the one hand, the Pujiang line RT21 integrated monitoring system is discussed on the other hand, the application structure and characteristics of Shanghai rail transit will lay a good technical foundation for the future use of this type of integrated monitoring system on the Shanghai rail transit network.
\end{abstract}

关键词: 综合监控;浦江线; 系统架构; 硬件

Keywords : integrated monitoring; Pujiang line; system structure; hardware

DOI : $10.36012 /$ etr.v2i7.2186

\section{1 引言}

上海浦江线 RT2 1 综合监控系统平台, 是中国国电南瑞 科技股份有限公司在 2017 年针对上海轨道交通浦江线运营 特点, 开发出来的大型综合监控软件平台。作为一套功能强 大模块化的城市轨道交通综合监控系统, 它集成联动了地铁 常用的通信、信号、机电与供电等系统。

\section{2 系统架构}

针对上海轨道交通浦江线的特殊运营模式(见图 1), 浦 江线 RT21 综合监控系统采用多层掌控, 意思是面对终端 层、车站层与 OCC 中心层的操作人员，给予其监管与控制
权, 依据控制权矛盾机制, 确保监控指令能可靠下达触发; 再 而分级管理, 面对不同操作人员的不同操作权限, 分配不同 监控画面及对应监控级别, 实现不同工作站的灵活配置。可 以说,通过多层掌控、分级管理,浦江线 RT21 综合监控系统 每一层的功能都相互独立, 又协调运行。

\section{1 软件框架}

浦江线 RT21 综合监控系统平台为分层式、模块化设计, 具体软件构架如图 2 所示。该系统平台应用 Linux 操作系 统, 优势在于其拥有成熟的平台模块, 如管理权限、图形工具 和报表工具等,在应用层承载各级子系统应用,如技术安防 CCTV 系统、SCADA 电力监控系统和广播系统等 (系统平台 


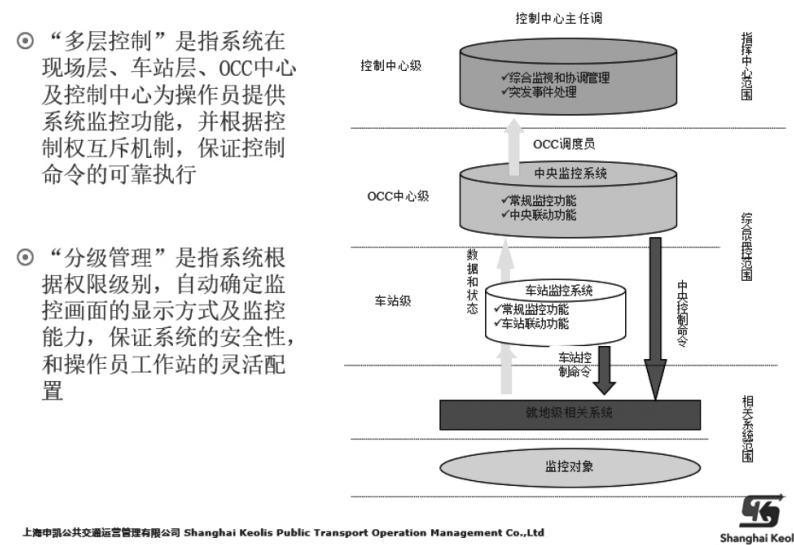

图 1 上海轨道交通浦江线的特殊运营模式

预留了信号 ATS 界面, 虽然上海浦江线有独立信号 ATS 系 统,但也说明了浦江线综合监控系统下属子系统接入的包容 与实用性, 完全涵盖轨道交通各级常用专业系统应用), 同时 平台兼具可扩展性强, 操作人员可针对不同需求, 进行合理 的编辑与修改。

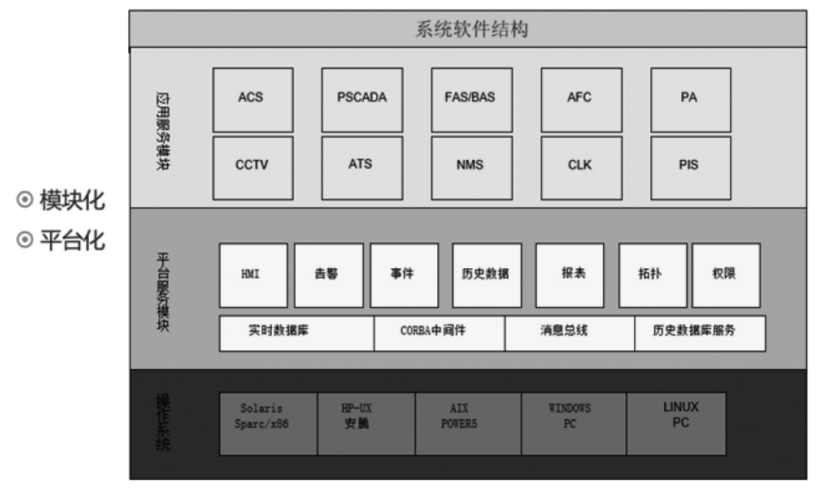

图 2 软件构架

\section{2 物理架构}

浦江线 RT21 综合监控系统平台以浦江线无人驾驶运营 工况为核心基础, 集成通信、供电和机电等城轨重要设备及 系统于一身。从物理框架上讲, 浦江线 RT2 1 综合监控系统 分为两级:一是中央级指的是浦江线 OCC 控制中心, 负责对 全线车站包括车辆段监视及控制; 二是浦江线下属 2 个集中 站东城一路站与三鲁公路站, 主要负责对本地正线车站状态 和实时监控数据进行收集和处理, 也可理解为正线车站人员 提供控制和监视权限。从基本硬件配置上讲, 无论是中央级 还是车站级硬件组成都包含 C306L 系列通信前置机、服务 器、交换机及工作站等终端设备, 且核心设备都具有尣余机 制保障。

纵观平台构成, 经过核心网络与其他车站相连接从而实
现车站正线及中心成为完整运行整体, 对外依靠 C306L 系列 通信前置机采集通信、信号、机电和供电等子系统应用。然后 综合监控核心网络把对应车站收集到的数据各自关联到车 站级实时服务器上 (东城一路站与三鲁公路站两套实时服务 器), 从而实现车站级综合监控系统的集成, 中央级也类似于 此, 只是需要汇总所有车站的数据。此外, 车站级综合监控平 台按每个车站配置了 IBP 盘(紧急后备盘), 如图 3 和图 4 所 示的系统物理构架图与拓扑图。

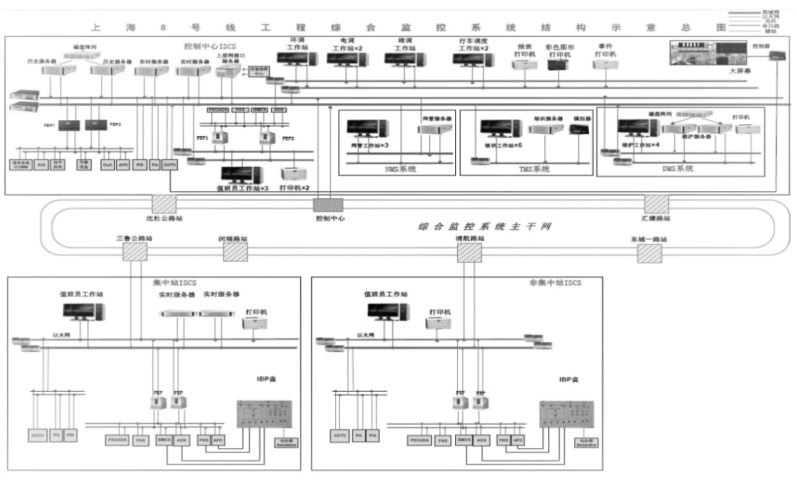

图 3 物理架构

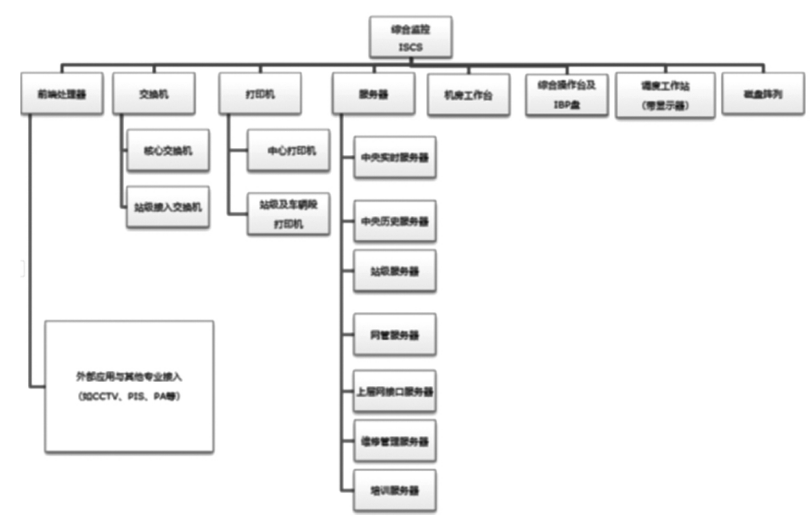

图 4 物理拓扑

\section{3 重要硬件组成}

\section{$3.1 \mathrm{C} 306 \mathrm{~L}$ 系列通信前置机}

浦江线 RT21 综合监控系统平台数据采集的核心枢纽是 基于南瑞自主研发的 C306L 系列通信前置机( FEP)。C306L FEP 通过机体载有的串行接口、网口及现场总线等通信介 质,按照特定的通信协议,将分布在不同位置的数据采集设 备所采集的实时数据进行集中收集,并形成统一的数据形式; 然后将这些实时数据根据需要向各自实时服务器端转发 ${ }^{[1]}$; 同 时 C306L FEP 可接收服务器端发出的各种形式的控制指令, 并转发给相应的数据采集设备或控制设备。 
主要接入设备 C306L FEP, 如图 5 所示。其主要技术参 数如下: (1) 16 个串行口, 兼容 RS23、RS422 和 RS485, 通信速 率为 300 57.6kbps; (2) 4 个 10/100M, 2 个 10/100/1000M 网 口, 自适应全双工 RJ45 以太网口; (3)内置 1 个(最多可扩展 至 3 个) 8 口(或 16 口)交换机模块; (4) 4 个 CAN 接口, 支持 BasicCAN 和 PeliCAN, 速率为 10 500kbps; (5) 1 个 DO 输出控 制点及 1 个 DI 输出控制点; (6)工作电源为 AC/DC 220V+ $20 \%$, 平均功率为 $<30 \mathrm{~W}$; (7)环境温度为 $-10 \sim 60^{\circ} \mathrm{C}$, 相对湿度 为 5\% 95\%, 环境气压为 $80 \sim 110 \mathrm{kPa}$ 。
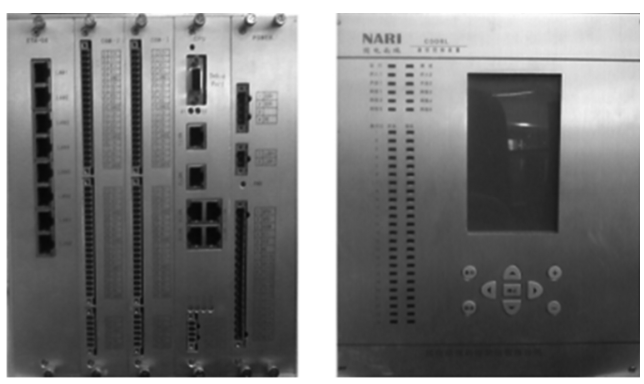

图 5 C306L 系列通信前置机

\section{2 磁盘阵列}

浦江线 RT21 综合监控系统采用 ORACLE 数据库管理 系统, 作为其综合监控系统关联的数据库。其中区别于车站 级, 浦江线 RT21 综合监控系统中央级的中央实时服务器 (午余)、历史服务器(午余) 、数据备份服务器都配置磁盘阵 列设备, 均采用与中央历史服务器原厂配套的同品牌产品 Oracle Pillar Axiom 600 阵列系统。磁盘阵列配置独立机柜, 拥有学余数据传输链路, 本系统的 1 套磁盘阵列采用 Oracle FS1-2 system, 主要配置为 2 个 Oracle FS1-2 控制器, 2 块 $16 \mathrm{~GB}$ 双口 HBA 卡, $4 \times 16 \mathrm{~GB}$ FC 接口, 24 个 300GB SAS 硬 盘, 磁盘阵列可存储一年以上的 ISCS 全线历史数据, 容量为 $8.4 \mathrm{~TB}$, 并可扩展。

\section{3 服务器}

浦江线 RT21 综合监控系统采用各类服务器配置, 如表 1 所示。

\section{4 工作站}

西门子 IPC 547E 工业计算机，工作站支持 GB 180302005 《信息技术 中文编码字符集》, 操作界面为简体中文界 面。配置要求: 基于 Windows 操作系统的工作站主机, 基本配 置 IPC 547E 工业计算机; Core i7-4770s 处理器, 4GB 内存, 2 个 500GB 硬盘, DVDRW, 3 个 10/100bps 以太网卡, 操作系统

\section{表 1 浦江线 RT21 综合监控系统采用各类服务器配置参数}

\begin{tabular}{|c|c|c|}
\hline \multirow{5}{*}{$\begin{array}{l}\text { RT21 综 } \\
\text { 合监控 } \\
\text { 服务器 }\end{array}$} & $\begin{array}{l}\text { 中央实 } \\
\text { 时服务 } \\
\text { 器 }\end{array}$ & $\begin{array}{l}2 \text { 台中央级实时服务器采用 Fujitsu M10-4S } \\
\text { server 和 Solaris 操作系统 }\end{array}$ \\
\hline & $\begin{array}{l}\text { 中央历 } \\
\text { 史服务 } \\
\text { 器 }\end{array}$ & $\begin{array}{l}2 \text { 台中央级历史服务器采用 Fujitsu M10-4S } \\
\text { server, 主要配置 } 2 \times S P A R C 64 \text { X 16-core } 3.0 \\
\text { GHz 处 理 器, 128GB DDR3 内 存, } 4 \text { 块 } \\
\text { 600GB SAS -2 硬盘, 8GB 光纤 HBA 卡, } \\
\text { Solaris 操作系统 }\end{array}$ \\
\hline & $\begin{array}{l}\text { 车 站 级 } \\
\text { 服务器 }\end{array}$ & $\begin{array}{l}6 \text { 座车站配置总共四台车站级服务器, 车站 } \\
\text { 级服务器采用 Fujitsu M10-4 server, 主要 } \\
\text { 配置 } 2 \text { 个 SPARC64 X 16-core } 2.8 \mathrm{GHz} \text { 处 } \\
\text { 理器, 64G DDR3 内存, } 2 \text { 块 600G SAS-2 硬 } \\
\text { 盘, Solaris 操作系统 }\end{array}$ \\
\hline & $\begin{array}{l}\text { 网管 服 } \\
\text { 务器 }\end{array}$ & $\begin{array}{l}1 \text { 台中央网管服务器采用 Fujitsu M10-4 } \\
\text { server, 主要配置 } 2 \text { 个 SPARC64 X 16-core } \\
2.8 \mathrm{GHz} \text { 处理器, 64G DDR3 内存, } 2 \text { 块 600G } \\
\text { SAS-2 硬盘, Solaris 操作系统 }\end{array}$ \\
\hline & $\begin{array}{l}\text { 上网接 } \\
\text { 口服务 } \\
\text { 器 }\end{array}$ & 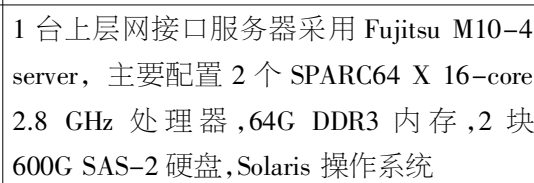 \\
\hline
\end{tabular}

基于64 位 Linux 操作系统的计算机工作站主机,工作站显示 器, Workstation display 采用 DELL E2216H 显示器。综合以上 工控机的优良硬件配置，保障了上海轨道交通浦江线 RT21 综合监控系统工作站电源可靠、计算处理能力迅捷及存储数 据安全等。

\section{5 交换机}

浦江线 RT21 综合监控系统的交换机分为两种, 分别是 核心交换机和站级接入交换机, 但无论哪种交换机配置, 都 保证成对互为呪余配置, 实现交换机尣余、端口乞余, 且要求 有足够的通道容量, 实现主备交换机无扰动切换。交换机皆 具有高效的多层交换性能和路由功能。以太网符合 IEEE 802.3 及 IEEE $802.3 \mathrm{u}$ 的规定。交换机具备虚拟局域网功 能, 支持 $802.1 \mathrm{p} 、 802.1 \mathrm{Q}$ 等标准协议。

核心交换机: 位于控制中心通信弱电机房, 共备用 2 套 核心交换机, 属于三层工业交换机, 型号为 MRD 子午线 TNM6000E。

站级接入交换机: 在各车站弱电机房设置 2 套交换机, 接入交换机三层工业交换机,MRD 子午线 TCC4100CW400G 用于 ISCS 系统服务器、工作站、打印机、各子系统 的网络接入。 


\section{4 浦江线 RT21 综合监控常见故障处理汇总}

\section{1 综合监控 FEP306 内进程故障}

当 ISCS 上无法操作某个子系统时候, 就需要从局部小 范围故障到全局故障去思考判断以缩小故障点的排查范围, 下面以实际例子举例说明。

2020 年 1 月 3 日 17:00, 控制中心调度报汇臻路消控室 ISCS 无法切换 CCTV, 检修人员开始排故。询问了调度控制 中心是否可以使用 ISCS 切换 CCTV 得到肯定答复后, 排除 了中央实时服务器故障的可能。之后询问临近车站即同一实 时服务器下所属的另外几个车站是否也出现相同故障现象, 得到未出现的答复后, 排除了由实时服务器下进程中断的可 能。之后检查 CCTV 本身自带的遥感键盘进行探头切换, 发 现切换也正常, 因此判断出该故障为 CCTV 与 ISCS 接口故 障, 于是在双方服务器上执行相关的必要操作以排除故障。

步骤一: 进入 CCTV 服务器执行相关指令操作以重启与 ISCS 的接口连接。

步骤二: 通过车站工作站远程连接到 ISCS 服务器, 找到 与汇臻路 CCTV 相关的进程。开始执行指令的关闭与启动操 作。当该指令重启完成之后, 再进行 CCTV 切换工作同时查 看此时指令的报文, 通过报文和实际能否切换判断该故障已 经修复。

\section{2 数据库及历史服务器报错}

由于浦江线 RT21 综合监控系统采用 ORACLE 系统作 为中央历史服务器与实时服务器关联的数据库 ${ }^{[2]}$, 如果发生 数据库与历史服务故障故障会影响中央级工作站正常工作， 下面以实际例子举例说明。

2020 年 4 月 25 日 13:15, 某调度工作站出现 CCTV、 PIS、广播等专业平台通信故障（子专业界面终端成红色状 态),重启工作站后弹出没有取到消息表,如图 6 所示告警界 面, 进入终端查看, 中央实时服务器均正常, 历史服务器出现 数据库连接失败, 具体如图 7 所示。

操作方法：

到 100.3/100.4 上面 cd/history, 发现有 I/O 错误, 代表和 磁盘阵列断开连接。确认磁盘阵列启用的情况下, 用 root 用 户 umount history, 再 mountall, 再试一下 mount history, 然后 再 stopdb, startdb, 恢复历史服务器与磁盘阵列之间连接, 故 障修复。另到 100.1 和 100.2 上面 showdb, 查看状态是否正

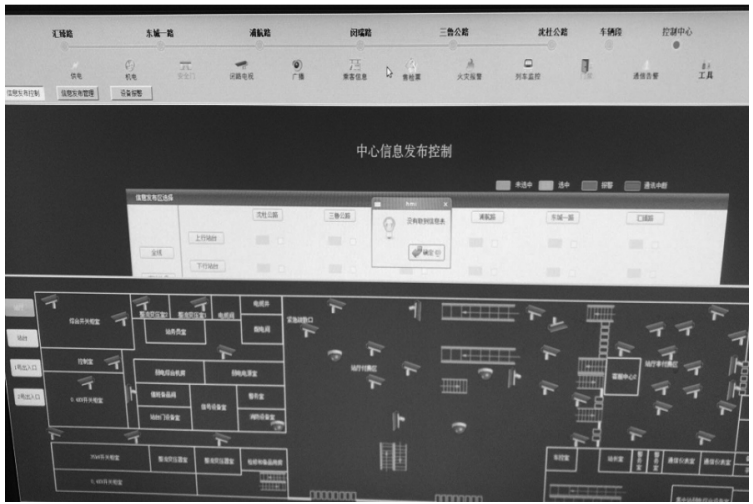

图 6 告警界面

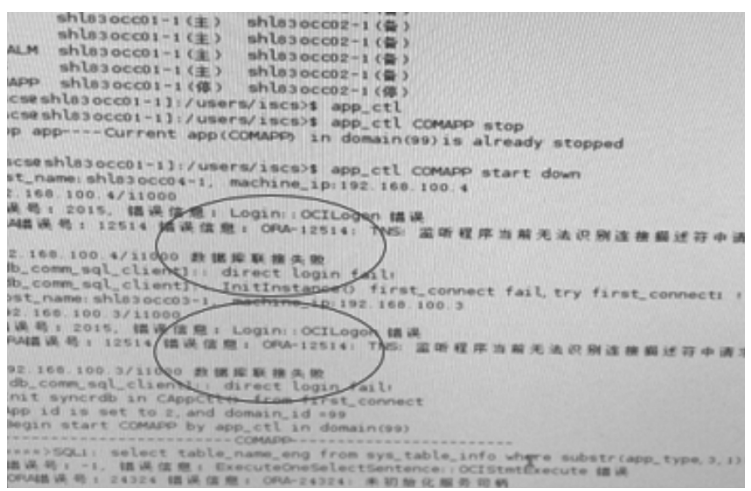

图 7 数据库连接失败

常。Iscs 用户下 startfv, 启动全景库。

\section{3 电力调度顺控卡片故障}

步骤一: 电力复式工作站上右击桌面, 打开终端; 输入地 址 telnet 192.168.100.1, 回车; 输人用户名与密码(此处密码 隐去); 输人 showdb, 查看是否有故障, 若无故障则代表数据 库正常, 若有故障联系以数据库软硬件方向修复。

步骤二: $c t l+c$ 退出 showdb 命令, 输入 procmng_show, 回 车查看 sequence_server 服务, 输入 procmng_del sequence_server;sequence_server, 回车, 重新杀掉该进程。尝试 是否成功, 若仍无法操作, 执行步骤三。

步骤三: 电力复式工作站上右击桌面, 打开终端; 输人地 址 telnet 192.168.100.1 地址, 回车; 输入用户名与密码(此处 密码隐去); 输人 app_ctl COMAPP stop, 回车; 等终端上代码 不跳动后输人 app_ctl COMAPP start down, 回车; 等终端上代 码不跳动; 打开新终端; 输人地址 telnet 192.168.100.2, 回车; 输人用户名与密码 (此处密码隐去); 输人 app_ctl COMAPP stop, 回车; 等终端上代码不跳动后输入 app_ctl COMAPP start down, 回车; 等终端上代码不跳动, 测验是否能进行顺控 停送电。 


\section{4 调度与车站工作站简单故障}

处理方法一：关闭 HMI 界面; 打开终端; cd /users/iscs/ rt21/data/pic ; rm *frb; rm *png; ; rm *svg。

处理方法二:关闭 HMI 界面; 打开终端; sys_ctl stop;cd / users/iscs/rt21/var/shm; ${ }^{*}$; cd /users/iscs/rt21/tmp; rm *。

\section{5 中央实时服务器宕机故障处理}

telnet 192.168.100.4(历史数据库 1), 输人用户名与密码 (此处密码隐去)

输人 startdb

telnet 192.168.100.3 (历史数据库 2), 输人用户名与密码 (此处密码隐去)

输人 startdb

telnet 192.168.100.1 (实时数据库 1), 输人用户名与密码 (此处密码隐去)

输人 sys_ctl start down

telnet 192.168.100.2( 实时数据库 2 ), 输人用户名与密码
（此处密码隐去）

输人 sys_ctl start down

键盘输人上述操作指令输入, 完成服务器启动。

\section{5 结语}

上海轨道交通浦江线于 2018 年 3 月 31 日开通试运营 至今已经平稳运行 3 年有余, 同时不知不觉浦江线 RT2 1 综 合监控系统也默默为这条无人驾驶运营线路保驾护航 3 个 寒暑, 正是由于其完善的专业集成性与良好的监控操作性, 兄现了上海浦江线运营从始至今一次次蜕变完善, 同时也为 建设未来的数字化城市轨道交通奠定了良好基础。

\section{参考文献}

[1] 葛轫,王成进,杨建旭,等.基于 RT21 的智能综合监控管理系统 [J].计算机系统应用,2014,23(12):77-81.

[2] 黄小平.RT21-ISCS 平台综合监控系统集成人机界面的设计 与实现[J].广东科技,2012,21(13):146-147+136.

\section{（上接第 4 页）}

\section{2 加强施工技术与人员管理}

由于建筑工程施工现场复杂, 对施工作业的专业性要求 较高, 使管理流程烦琐, 管理过程相对复杂。因此, 施工现场 的管理人员要熟悉施工流程, 严格管理每个流程所要求的施 工技术。

此外, 技术人员是进行施工操作、运用施工技术的主要 人员, 也是现场施工管理的对象, 对其进行严格管理, 有利于 提高其工作效率。首先, 要加强技术人员的专业技能的培训, 通过开展技能大赛、业务知识竞赛等活动, 提升其专业技能。 其次, 要明确技术人员的管理职责, 在保证完成本职工作的 基础上,提高其管理能力。再次, 要建立科学的管理考核制 度, 实行奖罚制度, 提高技术人员工作的积极性。最后, 技术 人员还要深人施工现场, 发现施工技术出现的问题要及时进 行处理和解决。

\section{3 加强监管工作}

施工质量对建筑工程施工管理而言, 是其管理的核心, 它直接影响着工程整体质量和经济效益。要解决施工质量这 个核心问题, 就需要管理人员配合监理方, 对施工质量严格 把控, 加强现场施工各个因素的管理, 通过对施工人员、材 料、设备、技术等进行全面管理, 做好质量检验管理工作。

\section{5 结语}

综上所述, 钢筋施工的质量对建筑工程的影响是巨大 的。在实际的生产施工过程中,一定要重视对相关技术的应 用, 不断地完善施工技术, 做好钢筋施工的质量控制, 进而为 建筑行业的发展和进步提供坚实的基础。

\section{参考文献}

[1] 付明雪. 建筑工程建设中现场施工管理研究[J]. 住宅与房地产. 2019(6):125. 